\title{
Globular cluster abundances: the role of asymptotic giant branch stars
}

\author{
Amanda I. Karakas \\ Mount Stromlo Observatory, Australian National University, Weston Creek ACT 2611, \\ Australia \\ email: akarakas@mso.anu.edu.au
}

\begin{abstract}
One of the more popular theories to account for the abundance anomalies in globular cluster stars is the 'self-pollution scenario,' where the polluters were a previous generation of intermediate-mass asymptotic giant branch (AGB) stars. This idea has proved attractive because: (i) the hot-bottom burning experienced by these objects qualitatively provides an ideal proton-capture environment to produce helium and convert $\mathrm{C}$ and $\mathrm{O}$ to $\mathrm{N}$, Ne to $\mathrm{Na}$ and $\mathrm{Mg}$ to $\mathrm{Al}$, and (ii) the slow winds from these stars allow their retention by the cluster's gravitational potential. New stellar yields from low-metallicity AGB models are presented and compared to abundances derived in globular clusters. We also discuss external pollution and inhomogeneouspollution models that use AGB stars as polluters. Current models of AGB stars cannot match all observational features of globular cluster stars. However, stellar modelling uncertainties are considerable and suggest AGB stars should not be ruled out just yet.
\end{abstract}

Keywords. stars: abundances, stars: AGB and post-AGB, globular clusters: general

\section{Introduction}

Star-to-star abundance variations of the light elements $\mathrm{Li}, \mathrm{C}, \mathrm{N}, \mathrm{O}, \mathrm{F}, \mathrm{Na}, \mathrm{Mg}$ and $\mathrm{Al}$ have been observed in every well-studied Galactic globular cluster (GC) to date (Kraft 1994; Gratton et al. 2004 and references therein), but are not found in field stars of the same metallicity (Gratton et al. 2000). Hence, these abundance patterns are somehow the result of the cluster environment. The variations of the elements follow a similar pattern: C-N, O-Na and $\mathrm{Mg}-\mathrm{Al}$ are anticorrelated (Shetrone 1996; Kraft et al. 1997; Cannon et al. 1998; Gratton et al. 2001; Cohen et al. 2005; Cohen \& Meléndez 2005). Furthermore, Smith et al. (2005) noted that stars in M4 showed evidence for a Na-F anticorrelation (an F spread was also found in NGC 6712; Yong et al. 2008a), while Pasquini et al. (2005) and Bonifacio et al. (2007) found a Na-Li anticorrelation in NGC 6752 and 47 Tucanae, respectively. The $\mathrm{Mg}-\mathrm{Al}$ anticorrelation is of particular interest, because from data for NGC 6752 (Yong et al. 2005), it is apparent that there is a larger spread in $\mathrm{Al}$ relative to $\mathrm{Fe}$, on the order of $\sim 1$ dex or more, but only a modest spread of $\sim 0.3$ dex in $\mathrm{Mg}$ relative to Fe. A further important test for any model is determining how well it can reproduce the $\mathrm{Mg}$ isotopic ratios observed in GC stars (e.g., in NGC 6752; Yong et al. 2003), which show a positive correlation between ${ }^{26} \mathrm{Mg}$ and $\mathrm{Al}$, and an anticorrelation between the dominant ${ }^{24} \mathrm{Mg}$ and $\mathrm{Al}$.

The abundances of iron-peak and neutron-capture elements do not show the same starto-star scatter nor do they vary with the light elements (Gratton et al. 2004, James et al. 2004; Yong et al. 2006, 2008b). There are exceptions noted in the literature, including the metal-rich cluster 47 Tuc (Wylie et al. 2006) and NGC 1851 (Yong et al. 2009). The other important exception is the massive cluster $\omega$ Centauri, whose age and metallicity spread, along with a rise in s-process element abundances with increasing $[\mathrm{Fe} / \mathrm{H}]$, suggests that 
it evolved very differently from other GCs and may have an extragalactic origin (Smith et al. 2000).

For a typical globular cluster, the key points are that oxygen has been destroyed in some stars by up to 1 dex or more, the CNO abundances are constant within uncertainties, and the $\mathrm{Na}$ and $\mathrm{Al}$ abundances are enriched relative to field stars of the same metallicity. The $\mathrm{Mg}$ isotopes in the 'polluted' stars are also nonsolar, with ${ }^{26} \mathrm{Mg}$ enriched at the expense of ${ }^{24} \mathrm{Mg}$, with no correlation between ${ }^{25} \mathrm{Mg}$ and Al. There is no evidence for large-scale variation of the neutron-capture elements.

The star-to-star abundance variations in C, N, O and Na have been observed in stars at or near the main-sequence turnoff (e.g., Gratton et al. 2001; Ramírez \& Cohen 2003), supporting the hypothesis that the GC stars did not produce their abundances in situ. Instead, a previous generation of stars polluted the atmospheres of stars we observe today or provided part of the material from which those stars formed (Cottrell \& Da Costa 1981). Because $[\mathrm{Fe} / \mathrm{H}]$ is roughly constant in stars in a given $\mathrm{GC}$, it has been assumed that the polluters were intermediate-mass AGB stars with initial masses between $\sim 4$ and $8 \mathrm{M}_{\odot}$ rather than supernovae, which produce Fe. The hot-bottom burning (HBB) experienced by these stars provides the hydrogen-burning environment (at least qualitatively) that can alter the abundances of the light elements via the CNO cycle, and the $\mathrm{NeNa}$ and $\mathrm{MgAl}$ hydrogen-burning chains. Another consequence is the production of a significant quantity of helium via the second dredge up as well as by HBB. Note that the slow winds from rapidly rotating massive stars have also been suggested as providing the hydrogenburning environment. This hypothesis is not discussed here, but we refer to Charbonnel (these proceedings) for further details.

The mass lost via the slow winds of AGB stars could, in principle, have been retained by the cluster from which new stars may have been born (Thoul et al. 2002). Detailed AGB models have so far mostly failed to match the observed abundance trends (e.g., Denissenkov \& Herwig 2003; Fenner et al. 2004; Karakas et al. 2006b). However, major uncertainties affect the model predictions and could leave room for an AGB solution (e.g., Ventura \& D'Antona 2005; Renzini 2008).

It is the AGB solution that will be discussed further in light of new stellar models of intermediate-mass AGB stars.

\section{Yields from asymptotic giant branch stars}

In AGB stars with initial masses $\gtrsim 4 \mathrm{M}_{\odot}$, depending on metallicity, $Z$, the base of the convective envelope becomes hot enough to sustain proton-capture nucleosynthesis (HBB). HBB can change the surface composition because the entire envelope is exposed to the hot-burning region a few thousand times per interpulse period. One important reason that intermediate-mass AGB stars could have polluted GCs is that these stars have relatively short lifetimes $(\tau \lesssim 100 \mathrm{Myr})$, compared to lower-mass AGB stars which have lifetimes of a few gigayears. Along with HBB, the repeated action of the third dredge up (TDU) will also change the surface composition. Following a thermal pulse, the convective envelope may move inward (in mass) to regions previously mixed by Heshell instabilities. This inward movement of the convective envelope is known as the TDU and is responsible for enriching the surface in ${ }^{12} \mathrm{C}$ and other He-burning products, as well as heavy elements produced by the slow-neutron-capture process.

Stellar nucleosynthesis predictions from low-metallicity, intermediate-mass AGB stars are available in the literature (e.g., Herwig 2004; Karakas \& Lattanzio 2007; Ventura \& D'Antona 2009). Here, we compare GC abundance trends using new AGB yields that are an update to the stellar yields presented in Karakas \& Lattanzio (2007). These new 

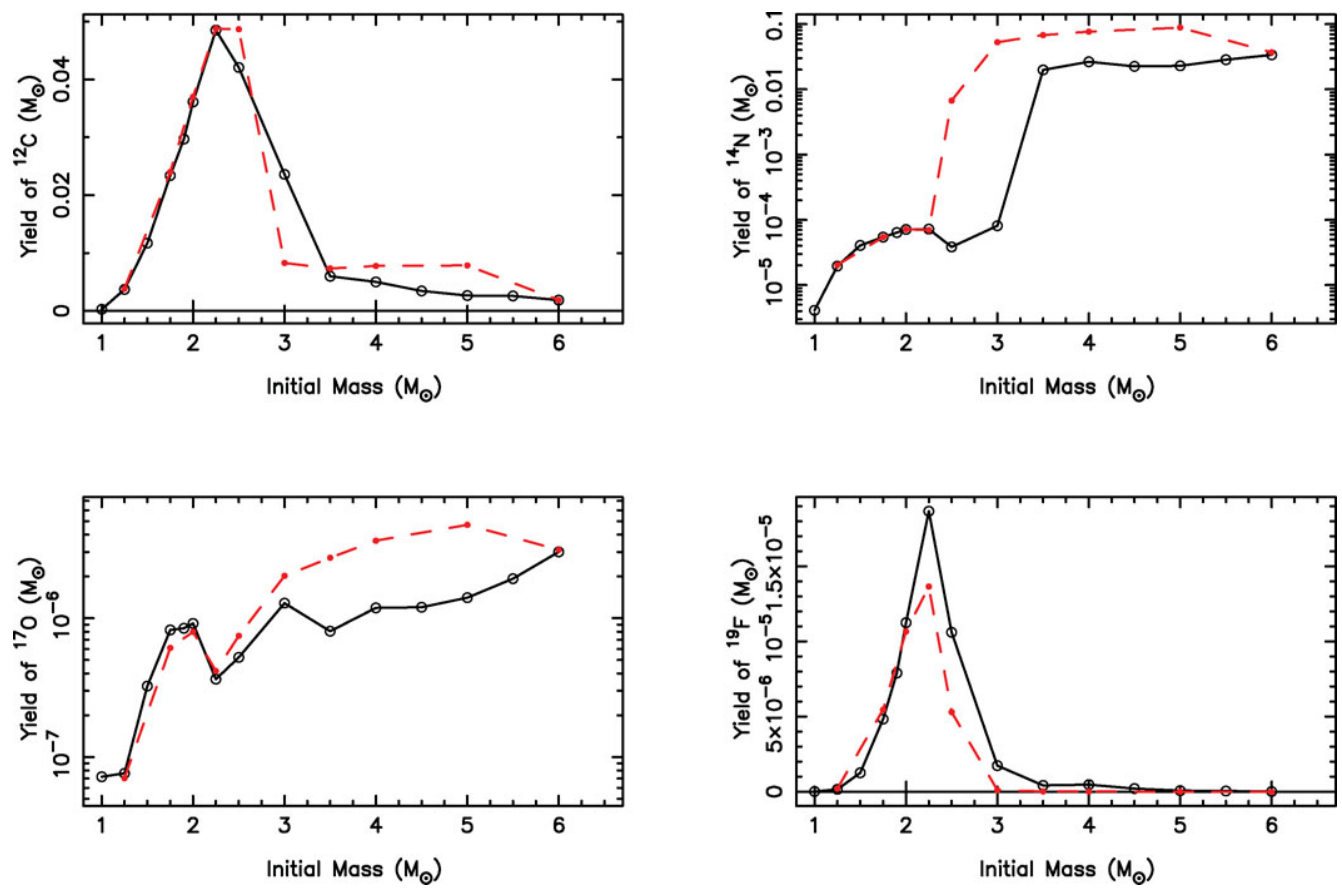

Figure 1. Yields of ${ }^{12} \mathrm{C},{ }^{14} \mathrm{~N},{ }^{17} \mathrm{O}$ and ${ }^{19} \mathrm{~F}$ (in $\mathrm{M}_{\odot}$ ) as a function of initial stellar mass from the $Z=0.0001$ models of Karakas (2009, open circles) compared to the yields from Karakas \& Lattanzio (2007, filled dots).

yields are discussed in detail in Karakas (2009); in summary, these models use updated reaction rates and provide yields from scaled-solar initial abundances for the $Z=0.008$ and 0.004 models.

In Figure 1 we show the yields of ${ }^{12} \mathrm{C},{ }^{14} \mathrm{~N},{ }^{17} \mathrm{O}$ and ${ }^{19} \mathrm{~F}$ as a function of initial stellar mass from the $Z=0.0001$ models of Karakas (2009) compared to the yields from Karakas \& Lattanzio (2007). The new yields of ${ }^{14} \mathrm{~N}$ from the intermediate-mass AGB models have been reduced, while the yields of ${ }^{12} \mathrm{C}$ are reasonably consistent with the previous calculations. Even with the reductions in ${ }^{14} \mathrm{~N}$, the total $\mathrm{CNO}$ expelled from these AGB models, regardless of mass, is increased compared to the initial amount by at least a factor of a few. If we take the $5 \mathrm{M}_{\odot}, Z=0.0001$ model as a representative example, the total CNO mass fraction at the surface increases by 2.5 dex during the AGB phase. This increase is driven by TDU mixing episodes, that mix primary ${ }^{12} \mathrm{C}$ from the He-burning shell to the envelope. Once there, HBB converts the ${ }^{12} \mathrm{C}$ to ${ }^{14} \mathrm{~N}$. It should be obvious that one of the important observational constraints found in GC stars has already been violated. Namely, if these clusters were polluted by a previous generation of $Z=0.0001$ AGB stars as modelled here, the CNO content of the most 'polluted' present-day stars (i.e., O depleted and Na enriched) should be higher than the stars with a 'normal' halo-like composition.

In Figure 2 we show the yields of ${ }^{23} \mathrm{Na}$ from the $Z=0.0001$ models of Karakas (2009) compared to the yields from Karakas \& Lattanzio (2007). Here we can see a huge reduction in the sodium yields, by a few orders of magnitude for the most massive models. There are two main reasons for this. First, the $\mathrm{NeNa}$ and $\mathrm{MgAl}$ chain proton-capture rates have been updated, and in particular the ${ }^{23} \mathrm{Na}(\mathrm{p}, \gamma){ }^{24} \mathrm{Mg}$ and ${ }^{23} \mathrm{Na}(\mathrm{p}, \alpha){ }^{20} \mathrm{Ne}$ rates from Hale et al. (2004) are faster than the rates we used previously (El Eid \& Champagne 


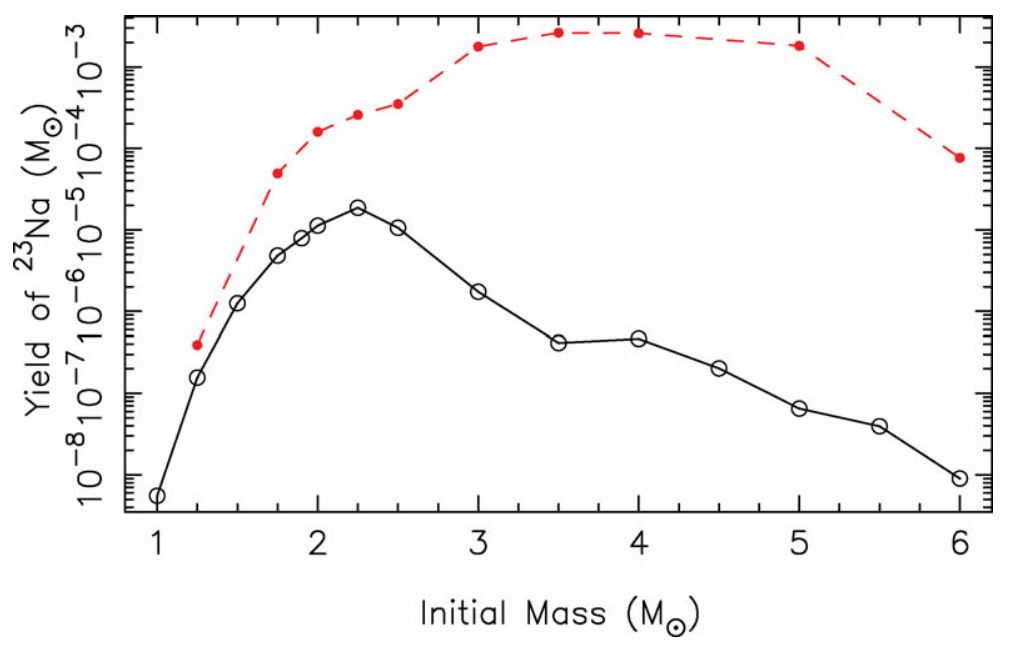

Figure 2. Yields of ${ }^{23} \mathrm{Na}$ (in $\mathrm{M}_{\odot}$ ) as a function of initial stellar mass from the $Z=0.0001$ models of Karakas (2009, open circles) compared to the yields from Karakas \& Lattanzio (2007, filled dots).

1994). Second, we have computed new intermediate-mass $Z=0.0001$ models with increased mass loss. These new models experience fewer thermal pulses and produce lower yields. It should be pointed out that the rates alone account for at least an order of magnitude decrease in sodium, and this can be seen at $2.5 \mathrm{M}_{\odot}$ where we are using the same stellar model as in Karakas \& Lattanzio (2007).

The self-consistent chemical evolution model of NGC 6752 described in Fenner et al. (2004) used AGB yields computed using an initial composition determined by a burst of Population III supernovae. In this model, the general trend of the $\mathrm{O}-\mathrm{Na}$ anticorrelation could be reproduced, however the AGB models produced too much $\mathrm{Na}$, and none of the models destroyed enough $\mathrm{O}$, except in the most massive $6.5 \mathrm{M}_{\odot}$ case. The $\mathrm{Mg}-\mathrm{Al}$ anticorrelation was not reproduced at all, because $\mathrm{Mg}$ and $\mathrm{Al}$ were produced together in the AGB models. Furthermore, both neutron-rich $\mathrm{Mg}$ isotopes, ${ }^{25} \mathrm{Mg}$ and ${ }^{26} \mathrm{Mg}$, are synthesized in the He-burning shells of AGB stars (e.g., Karakas et al. 2006a). For this reason, a correlation between these isotopes and $\mathrm{Al}$ was found, contrary to observations. The updated models of Karakas (2009) would not change these findings, because while less sodium was synthesized, the same trends between $\mathrm{Mg}$ and $\mathrm{Al}$ were observed. In summary, these AGB models cannot explain the abundance patterns observed in GC stars. First, the TDU mixes material from the He-burning shell to the envelope, resulting in increases in $\mathrm{CNO}$, and $\mathrm{Mg}$ and $\mathrm{Al}$. The TDU is also responsible for the large increases in Na: dredge up mixes (mostly primary) ${ }^{22} \mathrm{Ne}$ to the envelope and this is converted by proton capture to ${ }^{23} \mathrm{Na}$ through $\mathrm{HBB}$. Second, the amount of $\mathrm{O}$ destruction required ( 1 dex or more) is not found, except in the most massive AGB models.

Intermediate-mass AGB stars are subject to serious modelling uncertainties, in particular as regards the convection model used, the treatment of convective boundaries, and the mass-loss rate used during the AGB phase. Nuclear-reaction-rate uncertainties also strongly impact some isotopes (e.g., ${ }^{23} \mathrm{Na}$ and ${ }^{27} \mathrm{Al}$; see also Izzard et al 2007). In the next section, we examine other AGB model calculations in the literature. Can these do any better at reproducing the GC abundance patterns? 

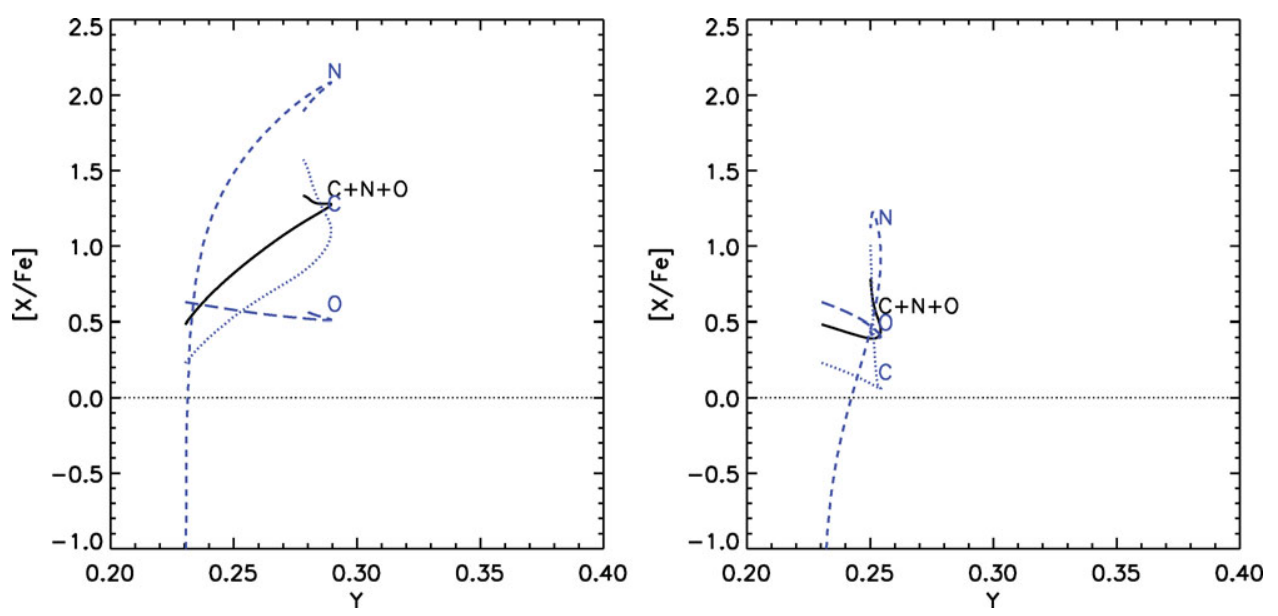

Figure 3. Temporal evolution of the $\mathrm{CNO}$ abundance $([\mathrm{CNO} / \mathrm{Fe}])$ in the cluster gas as a function of helium, $Y$, assuming a standard Salpeter initial mass function. (left) Results using yields from Fenner et al. (2004) and (right) yields from Ventura et al. (2002). We use the standard spectroscopic notation $[\mathrm{X} / \mathrm{Fe}]=\log (\mathrm{X} / \mathrm{Fe})-\log (\mathrm{X} / \mathrm{Fe})_{\odot}$. (From Karakas et al. 2006b.)

\section{Other AGB models in the literature}

Here we consider the intermediate-mass AGB models published by Ventura \& D'Antona (Ventura \& D'Antona 2009; and references therein). These stellar interior models are distinct from most other models because they use the 'full spectrum of turbulence' (FST) convection model instead of the mixing-length theory (MLT) of convection. In Ventura \& D'Antona (2005), the authors outlined the main differences between AGB models computed with FST versus the MLT. FST results in more efficient envelope convection, hence higher temperatures at the base of the convective envelope and consequently higher luminosities. Furthermore, the TDU is not predicted to be efficient. For this reason, little He-shell material is mixed into the envelope and this allows CNO to stay approximately constant, except at the lowest masses $\left(\approx 3 \mathrm{M}_{\odot}\right)$. Indeed, both $\mathrm{C}$ and $\mathrm{O}$ are destroyed in their most massive AGB models, contrary to the models of Karakas \& Lattanzio (2007) which produce both of these elements at the lowest metallicities $(Z=0.0001)$ as a result of efficient dredge up. However the lack of dredge up in the Ventura \& D'Antona models limits the amount of $\mathrm{Na}$ and $\mathrm{Al}$ that can be produced, so these authors have included a small amount of convective overshoot (e.g., Ventura \& D'Antona 2008).

In Figure 3 we show the results from Karakas et al. (2006b) of a chemical evolution model that uses the stellar yields from Ventura et al. (2002) in the right panel, compared to the yields from Fenner et al. (2004) in the left panel. In each panel, we show the evolution of helium on the $x$ axis versus $\mathrm{C}, \mathrm{N}$ and $\mathrm{O}$ on the $y$ axis. The combined $\mathrm{CNO}$ evolution is also shown. The main point of this figure is to show that the yields from Ventura et al. (2002) keep the CNO abundance approximately constant, to within 0.4 dex. In contrast, using the yields from Fenner et al. (2004), the CNO abundance increases by $\sim 0.8$ dex, well outside of observational uncertainties.

Owing to a higher efficiency of convection, the models of Ventura \& D'Antona are able to destroy more oxygen and, because there is less He-burning material, produce much less sodium (indeed, producing too little sodium has been a problem for these authors). However, overall it has been shown to be possible to reproduce the $\mathrm{C}-\mathrm{N}$ and 
O-Na anticorrelations using intermediate-mass AGB stars, even if the mass range that can reproduce these abundances is limited, depending on metallicity.

Reproducing the $\mathrm{Mg}-\mathrm{Al}$ anticorrelation and the behaviour of the $\mathrm{Mg}$ isotopes with $\mathrm{Al}$ has proven more difficult. There are two problems here: (i) producing enough Al by proton capture and (ii) reproducing the $\mathrm{Mg}$ isotopic abundances and behaviour with Al. For NGC 6752, the Al abundance has been shown to span more than 1 dex. Such large increases of $\mathrm{Al}$ are only possible in AGB models when using the upper limits of the ${ }^{26} \mathrm{Mg}(\mathrm{p}, \gamma){ }^{27} \mathrm{Al}$ and ${ }^{26} \mathrm{Al}(\mathrm{p}, \gamma){ }^{27} \mathrm{Si}$ reaction rates (e.g., Ventura \& D'Antona 2008). Furthermore, large increases in $\mathrm{Al}$ also require the combined operation of the TDU and $\mathrm{HBB}$, while without efficient dredge up $\mathrm{Al}$ production is limited. In regards to the $\mathrm{Mg}$ isotope ratios observed in NGC 6752, no AGB model has thus far successfully reproduced the observed spread, or behaviour with Al. This last constraint is a serious problem for all suggested polluters including the rotating massive stars.

\section{External pollution and inhomogeneous-pollution models}

Bekki \& Norris (2006) and Bekki et al. (2007) explored the idea that the abundance properties of the Galactic GCs were formed by 'external pollution.' In this framework, GCs are assumed to form in low-mass dwarf galaxies embedded in dark-matter subhaloes at very high redshifts. Field AGB stars of the dwarf galaxies then chemically influence the early GC-formation processes. GCs within a dwarf galaxy therefore can be formed from the mixture of (i) gas expelled from the field AGB stars formed earlier in the dwarf and (ii) the interstellar gas falling into the central region of the dwarf. The idea of external pollution is most attractive because it naturally provides an explanation for the large fraction of polluted stars without recourse to an implausible initial mass function. For example, for the self-pollution hypothesis to explain the large enhancements of helium $(Y \lesssim 0.4$ in mass fraction) postulated in massive clusters such as $\omega$ Cen and NGC 2808, an unusual initial mass function had been proposed by D'Antona \& Caloi (2004). Karakas et al. (2006) explored this idea further and found that a factor of 10 more intermediatemass AGB stars is required to obtain $Y \gtrsim 0.36$, depending on the fraction of primordial gas that is allowed to mix with the AGB ejecta.

Recently, Marcolini et al. (2009) explored the idea of an inhomogeneous-pollution chemical evolution model. This model depends on the existence of (i) a peculiar pre-enrichment phase in the GC's parent galaxy associated with very-low-metallicity Type II supernovae (SNe) and (ii) localized inhomogeneous enrichment from a single Type Ia SN and intermediate-mass AGB field stars. GC formation is assumed to take place within this chemically inhomogeneous region. The first low-mass stars to form are those with a 'polluted' composition (i.e., O depleted and $\mathrm{Na}$ enhanced), while 'normal' stars (i.e., O rich and $\mathrm{Na}$ depleted) are formed in a second stage when self-pollution from supernovae occurs and the inhomogeneous pollution from the previous phase is dispersed. This model naturally accounts for the low $[\mathrm{O} / \mathrm{Fe}]$ values of the most polluted stars, this is because such low $[\mathrm{O} / \mathrm{Fe}]$ values arise in the inhomogeneous region polluted by the initial SN Ia, which produces much more Fe compared to O. Note that the model is also able to maintain Fe approximately constant and to account for the $\mathrm{C}-\mathrm{N}, \mathrm{O}-\mathrm{Na}$ and $\mathrm{Mg}-\mathrm{Al}$ anticorrelations. One serious drawback that the model suffers is that the AGB yields (taken from Karakas \& Lattanzio 2007) still need to be adjusted to fit the data. The adjustments required include decreases in the yields of $\mathrm{C}$ and large increases in Al. From the discussion of the Ventura \& D'Antona models it is clear that such large adjustments are within the scope allowed by stellar modelling uncertainties. 


\section{Summary}

It has been postulated that low-metallicity, intermediate-mass AGB stars polluted the Galactic GCs while they were forming. This idea has proven attractive because these stars release slow winds that can be retained by the shallow potential well of GCs, they do not produce Fe and the HBB experienced by these stars provides the ideal hydrogenburning environment (at least qualitatively) that can alter the abundances of the light elements.

We have explored this idea using the latest AGB models available in the literature. We have compared the models to the observed abundance trends and conclude that AGB models cannot, in general, account for all observed chemical features of GC stars. The latest models from Karakas (2009) suffer from the same problem as discussed by Fenner et al. (2004), that is, material from the He-burning region increases the total CNO content of the AGB ejecta. Also, these models cannot destroy enough oxygen to satisfy the observational requirements. The AGB models from Ventura \& D'Antona provide a better match to most of the observed abundance trends, but still struggle to account for the large increases in $\mathrm{Al}$ and cannot reproduce the $\mathrm{Mg}$ isotopic abundances observed in NGC 6752.

However, major uncertainties affect the model predictions and could leave room for an AGB solution. Renzini (2008) argued convincingly that AGB stars are still the best candidates as polluters. He suggested major modifications to the AGB models including rapid ejection of the envelope following the second dredge up, which would allow little time for HBB and the TDU. Can these proposed modifications be tested against observations of the most luminous, oxygen-rich AGB stars in the Large and Small Magellanic Clouds (LMC and SMC, respectively)? The mean metallicity of the SMC is approximately the same as that of the stars in $47 \mathrm{Tuc}([\mathrm{Fe} / \mathrm{H}] \sim-0.7)$. If a generation of intermediate-mass AGB stars polluted 47 Tuc, then these polluters would have looked like the most massive, luminous AGB stars in the SMC. Observations of these stars suggest they are rich in Li and s-process elements (García-Hernández et al. 2009; Smith \& Lambert 1989). This evidence indicates that these luminous SMC giants have experienced HBB and the TDU. Given that neutron-capture elements are not correlated with light elements in most GCs, can these observations be used to rule out AGB stars as polluters?

\section{References}

Bekki, K. \& Norris, J. E. 2006, ApJ (Letters), 637, L109

Bekki, K., Campbell, S. W., Lattanzio, J. C., \& Norris, J. E. 2007, MNRAS, 377, 335

Bonifacio, P., Pasquini, L., Molaro, P., Carretta, E., François, P., Gratton, R. G., James, G., Sbordone, L., Spite, F., \& Zoccali, M. 2007, A\& A, 470, 153

Cannon, R. D., Croke, B. F. W., Bell, R. A., Hesser, J. E., \& Stathakis, R. A. 1998, MNRAS, 298,601

Cohen, J. G., Briley, M. M., \& Stetson, P. B. 2005, AJ, 130, 1177

Cohen, J. G. \& Meléndez, J. 2005, AJ, 129, 303

Cottrell, P. L. \& Da Costa, G. S. 1981, ApJ (Letters), 245, L79

D'Antona, F. \& Caloi, V. 2004, ApJ, 611, 871

Denissenkov, P. A. \& Herwig, F. 2003, ApJ (Letters), 590, L99

El Eid M. F. \& Champagne A. E. 1995, ApJ, 451, 298

Fenner, Y., Campbell, S., Karakas, A. I., Lattanzio, J. C., \& Gibson, B. K. 2004, MNRAS, 353, 789

García-Hernández, D. A., Manchado, A., Lambert, D. L., Plez, B., García-Lario, P., D’Antona, F. Lugaro, M., Karakas, A. I., \& van Raai, M. 2009, ApJ (Letters), 705, L31

Gratton, R. G., Sneden, C., \& Carretta, E. 2004, ARA\&A, 42, 385 
Gratton, R. G., et al. 2001, A\&A, 369, 87

Gratton, R. G., Sneden, C., Carretta, E., \& Bragaglia, A. 2000, A\& A, 354, 169

Hale, S. E., Champagne, A. E., Iliadis, C., Hansper, V. Y., Powell, D. C., \& Blackmon, J. C. 2004, Phys. Rev. C, 70, 045802

Herwig, F. 2004, ApJ, 605, 425

Izzard, R. G., Lugaro, M., Karakas, A. I., Iliadis, C., \& van Raai, M. 2007, A\&AA, 466, 641

James, G. et al. 2004, A\&A, 414, 1071

Karakas, A. I., Lugaro, M., Wiescher, M., Goerres, J., \& Ugalde, C., 2006, ApJ, 643, 471

Karakas, A. I., Fenner, Y., Sills, A., Campbell, S. W., \& Lattanzio, J. C. 2006, ApJ, 652, 1240

Karakas, A. I. \& Lattanzio, J. C. 2007, PASA, 24, 103

Karakas, A. I. 2009, MNRAS, submitted

Kraft, R. P. 1994, PASP, 106, 553

Kraft, R. P., Sneden, C., Smith, G. H., Shetrone, M. D., Langer, G. E., \& Pilachowski, C. A. 1997, AJ, 113, 279

Marcolini, A., Gibson, B. K., Karakas, A. I., \& Sánchez-Blázquez, P. 2009, MNRAS, 395, 719

Pasquini, L., Bonifacio, P., Molaro, P., François, P., Spite, F., Gratton, R. G., Carretta, E., \& Wolff, B. 2005, A\&BA, 441, 549

Renzini, A. 2008, MNRAS, 391, 354

Ramírez, S. V. \& Cohen, J. G. 2003, AJ, 125, 224

Shetrone, M. D. 1996, AJ 112, 1517

Smith, V. V. \& Lambert, D. L. 1989, ApJ (Letters), 345, L75

Smith, V. V., Suntzeff, N. B., Cunha, K., Gallino, R., Busso, M., Lambert, D. L., \& Straniero, O. 2000, AJ, 119, 1239

Smith, V. V., Cunha, K., Ivans, I. I., Lattanzio, J. C., Campbell, S., \& Hinkle, K. H. 2005, ApJ, 633,392

Thoul, A., Jorissen, A., Goriely, S., Jehin, E., Magain, P., Noels, A., \& Parmentier, G. 2002, $A \mathscr{E} A, 383,491$

Ventura, P. \& D'Antona, F. 2005, A\&A A, 431, 279

Ventura, P. \& D'Antona, F. 2008, $A \& A, 479,805$

Ventura, P. \& D'Antona, F. 2009, A\& A, 499, 835

Ventura, P., D’Antona, F., \& Mazzitelli, I. 2001, A\&\&A, 393, 215

Wylie, E. C., Cottrell, P. L., Sneden, C. A., \& Lattanzio, J. C. 2006, ApJ, 649, 248

Yong, D., Grundahl, F., Lambert, D. L., Nissen, P. E., \& Shetrone, M. D. 2003, A\&A, 402, 985

Yong, D., Grundahl, F., Nissen, P. E., Jensen, H. R., \& Lambert, D. L. 2005, A\& $A$, 438, 875

Yong, D., Aoki, W., Lambert, D. L., \& Paulson, D. B. 2006, ApJ, 639, 918

Yong, D., Meléndez, J., Cunha, K., Karakas, A. I., Norris, J. E., \& Smith, V. V. 2008, ApJ, 689, 1020

Yong, D., Karakas, A. I., Lambert, D. L., Chieffi, A., \& Limongi, M. 2008, ApJ, 689, 1031

Yong, D. Grundahl, F., D'Antona, F., Karakas, A. I., Lattanzio, J. C., \& Norris, J. E. 2009, ApJ (Letters), 695, L62 